\title{
PROSIST: SISTEMA INFORMÁTICO DE APOYO A LA PLANIFICACIÓN, MONITOREO Y CONTROL DE PROYECTOS DE I+D+I
}

\author{
ProSist: Informatics'system support to the planification, \\ monitoring and control of $R \& D \& l$ projects
}

\section{EPISTEMUS}

ISSN: 2007-8196 (electrónico)

ISSN: 2007-4530 (impresa)

Emilio Enrique Lucas López ${ }^{1}$

Neysi lleana León Pupo ${ }^{2}$

María Isabel Castellanos Domínguez ${ }^{3}$

Recibido: 15 de septiembre de 2018,

Aceptado: 29 de noviembre de 2018

Autor de Correspondencia:

Neysi lleana León Pupo

Correo:nleon@uho.edu.cu

\section{Resumen}

Los proyectos constituyen la célula básica para la organización, ejecución, financiamiento y control de las actividades de investigación científica, desarrollo tecnológico e innovación. Se requiere una adecuada gestión de la información de los proyectos de I+D+i de la Universidad de Holguín, lo cual favorece directamente la planificación, monitoreo y control que se lleva por parte de los asesores de proyectos de la Dirección de Ciencia y Tecnología e Innovación. Para satisfacer esta necesidad durante el curso 2016-2017 un grupo de investigadores se dieron la tarea de informatizar este proceso. En esta investigación se aporta la aplicación web ProSist, desarrollado en el lenguaje de programación PHP y el framework de desarrollo web Symfony2 principalmente. La combinación de estas tendencias y tecnologías permitió obtener un sistema informático que satisface las necesidades que lo originaron y de gran utilidad para la gestión de la información de proyectos.

Palabras clave: proyectos I+D+i, aplicación web, planificación, monitoreo, control

\section{Abstract}

The projects constitute the basic cell for the organization, execution, financing and control of the activities of scientific research, technological development and innovation. For this reason, an adequate management of the information of the R\&D\&I projects of the University of Holguin is required, which directly favors the planning, monitoring and control that is carried out by the project advisors of the Directorate Science and Technology. To meet this need during the 2016-2017 academic year, a group of researchers took on the task of computerizing this process. In this research the ProSist web application, developed in the PHP programming language and the Symfony web development framework is contributed. The combination of these trends and technologies allowed to obtain a computer system that satisfies the needs that originated and of great utility for the information management of projects.

Keywords: R\&D\&I projects, web application, planning, monitoring, control

Universidad de Holguín. Avenida XX Aniversario. Carretera a Guardalavaca, Holguín. CP 80 100. elucasl@uho.edu.cu Universidad de Holguín. Avenida XX Aniversario. Carretera a Guardalavaca, Holguín. CP 80 100. nleon@uho.edu.cu 2 


\section{INTRODUCCIÓN}

La actividad de gestión de proyectos en Cuba se rige por regulaciones relativas a la organización del Sistema de la Ciencia Cubana, expresadas en la Resolución 44/2012 del Ministerio de Ciencia, Tecnología y Medio Ambiente (CITMA). Este reglamento tiene como objeto normar el proceso de elaboración, aprobación, planificación, ejecución y control de los programas y proyectos de Ciencia, Tecnología e Innovación (CTI) en todos los niveles de la organización económica del país (Ministerio de Ciencia Tecnología y Medioambiente,2012). En el año 2014 se crean las Indicaciones Metodológicas (Ministerio de Ciencia Tecnología y Medioambiente,2014) con el objetivo de complementar la implementación de la resolución antes mencionada. Todas las instituciones del país y específicamente las universidades deben crear mecanismos que les permitan ejecutar esta resolución.

En la Universidad de Holguín (Laguna Cruz,2017) la gestión de proyectos se concibe como el eje fundamental del proceso investigación. El subproceso de gestión de proyectos es central y a partir de él se generan los otros subprocesos como la gestión de publicaciones, eventos y premios. Para que surja un nuevo proyecto deben coincidir la generación de una idea que responda a una prioridad ya sea internacional, nacional o de la propia institución (León Pupo,2016). La Dirección de Ciencia, Tecnología e Innovación (DCTI) se encarga de la gestión del proceso de investigación y tiene entre sus objetivos fundamentales asegurar que todas las acciones de investigación se sostengan en proyectos y el perfeccionamiento constante de la gestión de la CTI de la universidad asegurando el desarrollo de la planificación de la actividad de forma proactiva y sistémica y el control de modo sistemático (León Pupo,2016), (Ortíz Pérez,2014).

En la actividad de proyectos, estudios empíricos realizados a través de la experiencia propia, análisis de informes y bibliografías científicas, entrevistas a directivos, investigadores y jefes de proyectos, además de la observación directa, han podido detectar las siguientes deficiencias:

$\checkmark$ Duplicidad de información. Cuando un resultado o una salida de estos (publicaciones, premios, propiedad intelectual) pertenecen a investigadores de diferentes áreas se reportan por cada una de ellas.

$\checkmark$ No estandarización de la información. Aun cuando se envían indicaciones específicas para la elaboración de los planes y balances y sus reportes estadísticos, no se entregan en el formato requerido lo que hace difícil la tarea de la DCTI al integrar los informes.

$\checkmark$ Dificultades para gestionar la información de los

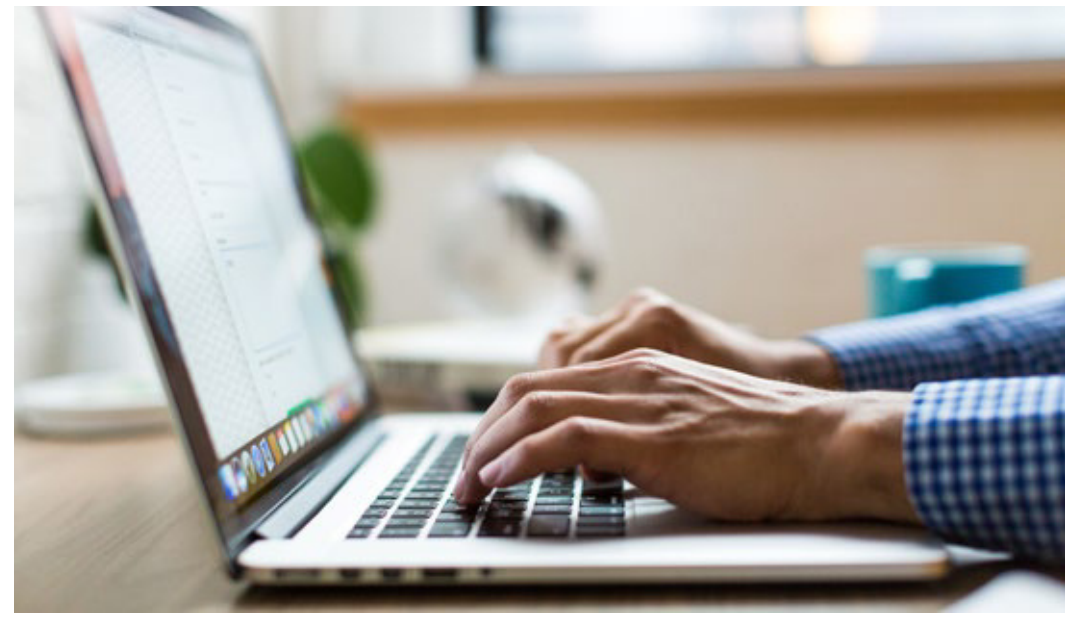

proyectos, así como la integración de la misma, debido a que se realiza en libros Excel por varios especialistas lo que significa un gran número de versiones desactualizadas.

- La información brindada de los investigadores en los perfiles de proyectos y los documentos de los expedientes no siempre está completa o actualizada. Nombres escritos de manera diferente, categorías docentes desactualizadas y otras.

Existen novedosas opciones de software de código abierto (open source) para la gestión de proyectos y tareas entre los cuales se encuentran: Taiga.io ${ }^{1}$, TeamBox ${ }^{2}$, LibrePlan ${ }^{3}$, OpenProject ${ }^{4}$, Agilefant ${ }^{5}$, Mantis Bug Tracker6. Estos sistemas van dirigidos a la gestión de actividades por participante en función del tiempo, las cuales serían muy útiles para la planificación y organización del equipo de trabajo en un determinado proyecto.

Sin embargo, no permiten un monitoreo general por parte de los asesores de proyectos, que es lo que se requiere en esta investigación. Se requiere además que puedan gestionar todos los modelos de la Resolución 44/2012 del CITMA.

Debido a lo anterior se demuestra la necesidad de desarrollar un nuevo sistema informático (ProSist) que dé respuesta a estas necesidades.

\section{MATERIALES Y MÉTODOS}

\section{El subproceso de gestión de proyectos en la UHo.}

El proceso de gestión de proyectos (Figura 1) es central y a partir de él se generan los otros subprocesos. Para que surja un nuevo proyecto deben coincidir la generación idea con la existencia de una prioridad ya sea internacional, nacional o de la propia institución. Las

$1 \mathrm{https} / / /$ taiga.io/

2 https://redbooth.com/es/

3 https://www.libreplan.org/

4 https://www.openproject.org/

5 https://www.agilefant.com/product/

6 https://www.mantisbt.org/ 
prioridades internacionales surgen de convocatorias de fuentes de financiamiento. Las prioridades nacionales aparecen a partir de las convocatorias a programas nacionales establecido por el CITMA; los programas territoriales como una contextualización a las necesidades del territorio; necesidades específicas de desarrollo de una empresa; y la propia universidad (León Pupo,2016).

A partir de la firma del contrato el equipo de proyecto debe cumplir con el cronograma y los resultados de investigación previstos. Los resultados se plasman en informes parciales y finales los que son objeto de evaluación por los CCA antes de entregar a los financistas.

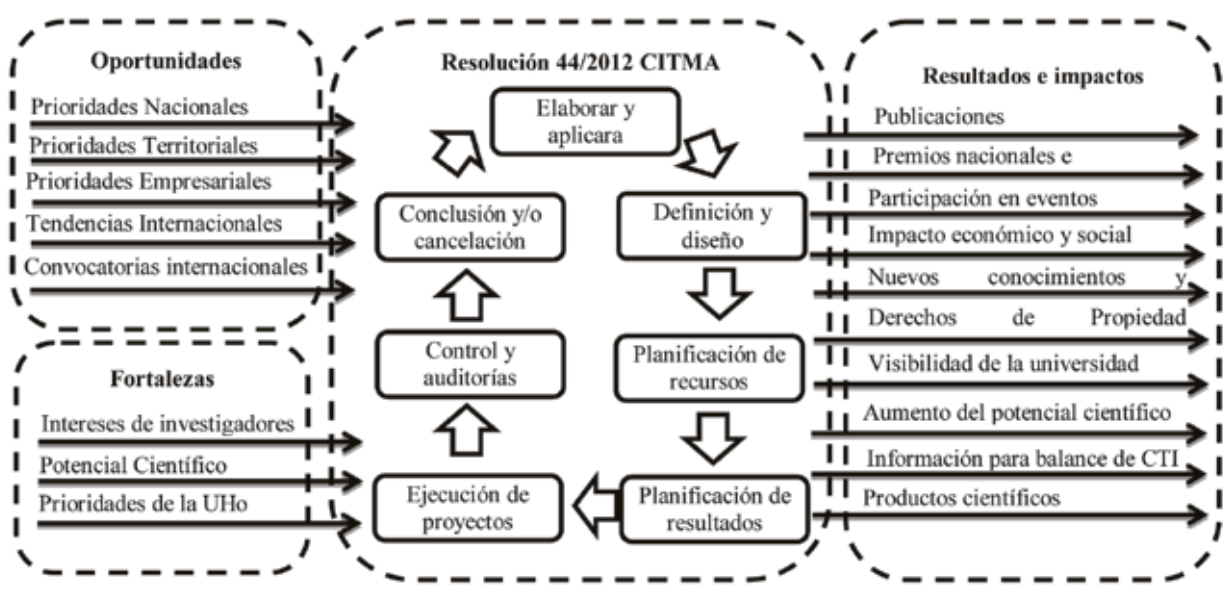

ENTRADAS

TRANSFORMACIONES

SALIDAS

Figura 1 proceso Modelo de proceso de Gestión de proyectos de la UHo.

Fuente: (León Pupo, 2016)

\section{PLANIFICACIÓN, MONITOREO Y CONTROL DE PROYECTOS DE I+D+I}

\section{Planificación}

Planificar significa estudiar anticipadamente los objetivos y acciones a desarrollar, y sustentar los actos en métodos, plan o lógica estableciendo los objetivos del proyecto y definiendo los procedimientos adecuados para alcanzarlos (Stoner,1996). El plan de proyectos del año siguiente se realiza desde el mes de abril a partir de los proyectos aprobados en convocatorias de programas nacionales, con empresas y convocatorias institucionales. Todos los proyectos deben ser avalados por el Consejo Científico Asesor (CCA) del área o la UHo.

\section{Monitoreo}

El monitoreo es el seguimiento que se realiza durante la ejecución de un programa o proyecto. Es un instrumento, de gestión, que permite revisar en forma periódica los aspectos sustantivos de los programas o proyectos, para optimizar sus procesos, resultados e impactos. Constituye un mecanismo indispensable para la gestión administrativa y estratégica (Stoner,1996).

Al final de cada semestre (junio, diciembre) el jefe de proyecto realiza el informe del proyecto que incluye una certificación de actividades acorde a lo planificado en el período. Posteriormente el CCA de la entidad ejecutora principal analiza el cumplimiento de las actividades que culminan dentro del período y emite el modelo de Informe semestral del proyecto, el cual es firmado por el director de dicha entidad y entregado a los asesores de proyecto de la DCTI UHo. En el caso de los proyectos asociados a programas nacionales (PAPN) y los no asociados a programas (PNAP) deben ser enviados además a los secretarios de los programas nacionales (Ministerio de Ciencia Tecnología y Medioambiente,2014).

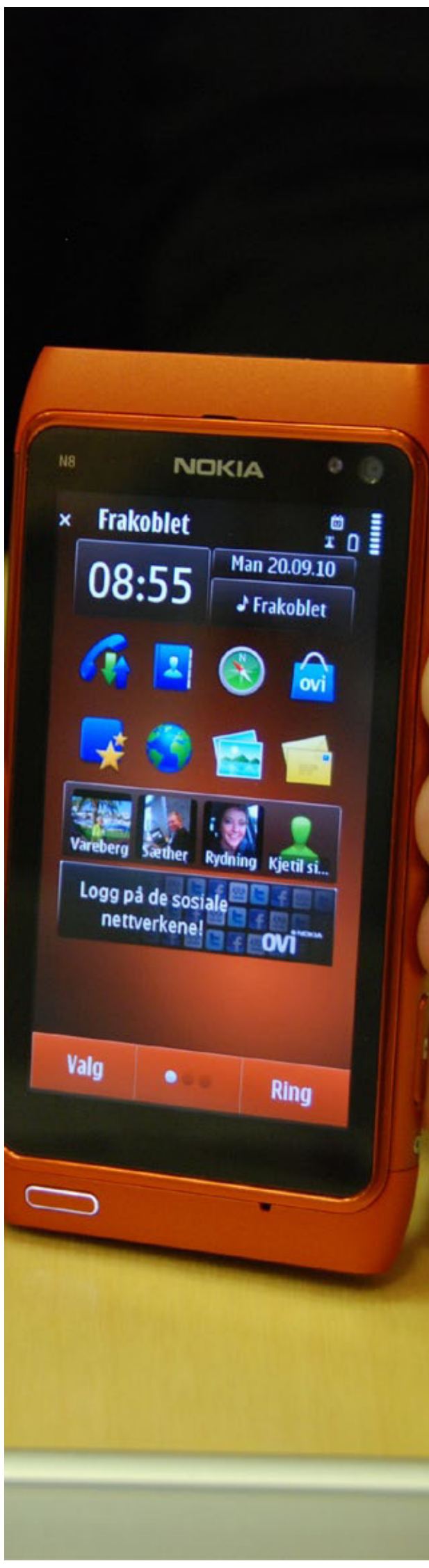




\section{Control}

El control es un proceso sistemático mediante el que se fijan los niveles de actividad, se diseñan los sistemas de retroalimentación de la información para el análisis del avance hacia los objetivos, y se reúnen los elementos requeridos para la toma de decisiones que conduzcan al cumplimiento de las metas de la organización (Stoner,1996).

El control de proyectos se realiza en tres momentos diferentes el control de calidad; el control de ejecución que se puede manifestar en auditorias de organizaciones autorizadas a ejercer funciones de control; y el finalmente el control de salida resultados por sus diferentes etapas son sometidos a la evaluación del CCA del área correspondiente y de la Universidad. Las principales formas de control de los proyectos son: las evaluaciones trimestrales que realiza el Consejo Científico Asesor de la entidad ejecutora principal; las evaluaciones semestrales y anuales que realiza el Grupo de Expertos y las evaluaciones no periódicas que realizan las entidades que gestionan los proyectos, así como los que dirigen los programas y el CITMA.

Las evaluaciones no periódicas se realizan, al menos una vez al año. Se efectúan, por las autoridades facultadas para ejercer el control (asesores de gestión de proyectos, directivos de la DCTI UHo o funcionarios del CITMA), mediante diferentes modalidades como: visitas in situ (visitas integrales a las áreas orientadas y dirigidas por la Vicerrectoría de Integración y Gestión Estratégica), entrevistas a los participantes, entrevistas a los clientes, realización de talleres, entre otras. Los resultados de las evaluaciones no periódicas se documentan y forman parte del expediente del programa o del proyecto.

En las visitas in situ se controlan, en detalle, los aspectos científico-tecnológicos, económicos y financiero y el cumplimiento de los requerimientos de la defensa. Las entrevistas a los participantes y clientes permiten profundizar aspectos específicos relacionados con la ejecución, la introducción de los resultados y la realización de las conciliaciones requeridas (Ministerio de Ciencia Tecnología y Medioambiente,2014).

Tendencias y tecnologías actuales empleadas en el sistema propuesto

Para el desarrollo de sistemas informáticos, existen diversas herramientas y tecnologías. Para la implantación exitosa de los mismos se debe asegurar que las escogidas sean las más adecuadas según el problema a resolver. A continuación se describen las que fueron seleccionadas para dar cumplimiento al objetivo de la investigación, precisando sus principales características y ventajas.

\section{Arquitectura Cliente-Servidor}

La arquitectura Cliente-Servidor es un modelo para el desarrollo de "sistemas distribuidos entre múltiples procesadores donde hay clientes que solicitan servicios y servidores que los proporcionan" (Valle,2005). Este modelo es el que más se ajusta a las necesidades del sistema propuesto por las características del flujo de información identificado dentro de la organización y la infraestructura tecnológica que posee, la distribución espacial de los entes que actúan, debido a que la UHo cuenta con cuatro sedes distribuidas por toda la capital provincial y 13 Centros o Fililaes Universitarias (CUM /FUM) en toda la provincia. Además, la necesidad de acceso a los datos desde cualquier sitio de la red y de una eficiente gestión de la información.

\section{Aplicación Web}

Una aplicación Web utiliza un sitio Web como fachada para mostrar una aplicación alojada en un servidor, resulta importante destacar que las aplicaciones Web poseen características como el procesamiento de solicitudes HTTP (Hiper Text Transfer Protocol) dinámicamente, esta característica es empleada por las organizaciones para representar sus propias lógicas de negocio (Martín, A.R,2005).

Se decidió implementar el sistema informático como Aplicación Web, debido a la necesidad de que la información pueda ser accedida desde cualquier punto en la red de la universidad en los diferentes locales y depender solo de un navegador Web como cliente para hacer uso de ella, sin necesidad de contar con una máquina computadora con elevados recursos de procesamiento o una gran capacidad de memoria física. Además, el mantenimiento y las actualizaciones que se le hagan a la aplicación se realizarían solo en la máquina servidor.

\section{Tecnologías de servidor web. Apache}

Un servidor Web es un programa que está en ejecución $y$ en espera de solicitudes de forma continua. Consta de un intérprete de HTTP, el cual se mantiene a la espera de peticiones y responde con el contenido solicitado. El cliente se encarga de interpretar el código y lo exhibe en pantalla (Murray,2017).

El servidor Apache HTTP, más conocido como Apache, es un servidor web HTTP de código abierto para la creación de páginas y servicios web. Es un servidor multiplataforma, gratuito, muy robusto y que se destaca por su seguridad y rendimiento. Apache tiene como principales características: es un software de código abierto, y completamente gratuito, posee una alta aceptación en la red y muy popular, esto hace que muchos programadores de todo el mundo contribuyen constantemente con mejoras, que están disponibles para cualquier persona que use el servidor web y que Apache se actualice constantemente, es multi-plataforma y tiene la capacidad de manejar más de un millón de visitas/día (Gandhi,2002).

\section{Sistemas gestores de bases de datos. MySQL}

Sistema Gestor de Bases de Datos (SGBD) es un software que proporciona servicios para la creación, el almacenamiento, el procesamiento y la consulta de la información almacenada en base de datos de forma segura 
y eficiente. Un SGBD actúa como un intermediario entre las aplicaciones y los datos, oculta la representación interna de los datos y ofrece un conjunto de funciones de más alto nivel (Cabello,2010).

MySQL, es un sistema de administración relacional de bases de datos. Una base de datos relacional archiva datos en tablas separadas en vez de colocar todos los datos en un gran archivo. Esto permite velocidad y flexibilidad. Las tablas están conectadas por relaciones definidas que hacen posible combinar datos de diferentes tablas sobre pedido. Es un sistema cliente/servidor, por lo que permite trabajar como servidor multiusuario y de subprocesamiento múltiple, o sea, cada vez que se crea una conexión con el servidor, el programa servidor establece un proceso para manejar la solicitud del cliente, controlar así el acceso simultáneo de un gran número de usuarios a los datos y asegurar el acceso a usuarios autorizados solamente. Este gestor de bases de datos aprovecha la potencia de sistemas multiprocesador, gracias a su implementación multihilo, soporta gran cantidad de tipos de datos para las columnas, dispone de API's en gran cantidad de lenguajes (C, C++, Java, PHP, etc.), posee una gran portabilidad entre sistemas, soporta hasta 32 índices por tabla, y su gestión de usuarios y contraseñas, mantiene un muy buen nivel de seguridad en los datos (Nixon, R,2014).

\section{Lenguaje de Programación. PHP}

PHP (Hypertext Pre-processor) es un lenguaje de programación usado normalmente para la creación de contenido para sitios web con los cuales se pueden programar las páginas HTML (HyperText Markup Language) y los códigos de fuente. Es un lenguaje interpretado usado para la creación de aplicaciones para servidores o creación de contenido dinámico para sitios web. PHP es un lenguaje multiplataforma, con una capacidad de conexión con la mayoría de los manejadores de base de datos que se utilizan en la actualidad, lee y manipula datos desde diversas fuentes, incluyendo datos que pueden ingresar los usuarios desde formularios HTML, tiene capacidad de expandir su potencial al utilizar una enorme cantidad de módulos (llamados extensiones), es libre por lo que se presenta como una alternativa de fácil acceso para todos, permite las técnicas de Programación Orientada a Objetos, permite crear los formularios para la web y tiene una biblioteca nativa de funciones sumamente amplia e incluida (Heurtel, O,2015), (Olsson, M,2016).

\section{HTML}

HTML y es el lenguaje que todos los programas navegadores usan para presentar información en la World Wide Web (WWW). Este es un lenguaje muy sencillo que se basa en el uso de etiquetas. HTML5 provee básicamente tres características: estructura, estilo y funcionalidad. HTML5 es considerado el producto de la combinación de HTML, CSS (Cascading Style Sheets) y Javascript. Estas tecnologías son altamente dependientes y actúan como una sola unidad organizada bajo la especificación de HTML5 (Gauchat, J.D,2017). Más allá de esta integración, la estructura sigue siendo parte esencial de un documento. La misma provee los elementos necesarios para ubicar contenido estático o dinámico y es también una plataforma básica para aplicaciones. Además, la estructura debe proveer forma, organización y flexibilidad.

\section{Javascript}

Javascript es un lenguaje de programación que surgió con el objetivo inicial de programar ciertos comportamientos sobre las páginas web, respondiendo a la interacción del usuario y la realización de automatismos sencillos. En ese contexto nació como un "lenguaje de scripting" del lado del cliente. Las necesidades de las aplicaciones web modernas y el HTML5 han provocado que el uso de Javascript haya llegado a unos niveles de complejidad y prestaciones tan grandes como otros lenguajes de primer nivel. Además, en los últimos años Javascript se está convirtiendo también en el lenguaje "integrador" (Hoffman, S.,2016).

\section{Framework Symfony 2}

Un framework se puede considerar como una aplicación genérica incompleta y configurable a la que se puede añadir las últimas piezas para construir una aplicación concreta. El framework es una herramienta estratégica para apoyar a los arquitectos de software en la toma de la mejor decisión para el desarrollo de aplicaciones web como son: acelerar el proceso de desarrollo, reutilizar código ya existente y promover buenas prácticas de desarrollo como el uso de patrones (Gutiérrez,2014).

Symfony2 está desarrollado completamente en PHP 5.3 y es compatible con la mayoría de gestores de bases de datos, como MySQL, PostgreSQL, Oracle y Microsoft SQL Server. Se puede ejecutar tanto en plataformas *nix (Unix, Linux, etc.) como en plataformas Windows. Además es un framework libre.

En el desarrollo del sistema propuesto se utilizó Symfony2 puesto que es un framework completo diseñado para optimizar el desarrollo de las aplicaciones web basado en el patrón Modelo Vista Controlador. Este framework separa la lógica de negocio, la lógica de servidor y la presentación de la aplicación web. Proporciona varias herramientas y clases encaminadas a reducir el tiempo de desarrollo de una aplicación web compleja. Además, automatiza las tareas más comunes, permitiendo al desarrollador dedicarse por completo a los aspectos específicos de cada aplicación (Armand,2014).

\section{Metodologías de desarrollo de software}

Es el conjunto de filosofías, fases, procedimientos, técnicas, reglas, herramientas y un soporte documental que ayuda a los desarrolladores a realizar aplicaciones informáticas con el fin de hacerlo más predecible y eficiente. Dentro de las principales metodologías tradicionales se 
encuentra RUP y en la categoría de ágiles entra Iconix (Rosenberg,2005).

ICONIX es un proceso simplificado en comparación con otros procesos más tradicionales, que unifican un conjunto de métodos de orientación a objetos con el objetivo de abarcar todo el ciclo de vida de un proyecto. Para el desarrollo del sistema informático propuesto se seleccionó Iconix.

\section{RESULTADOS Y DISCUSIÓN}

ProSist: Sistema Informático de apoyo al proceso de planificación, monitoreo y control de proyectos de $\mathrm{I}+\mathrm{D}+\mathrm{i}^{1}$

\section{Actores del sistema}

Los actores representan el rol que desempeña una o varias entidades que deben intercambiar información con el sistema, el actor es llamado comúnmente usuario, aunque le es dado el nombre del rol específico que desempeña en el sistema. Es importante destacar que el orden en que aparecen los actores en la Tabla 1 corresponde con los privilegios que tienen dentro del sistema y la herencia que tienen entre ellos también se comporta en el modo que estos aparecen.

Tabla 1 Actores del sistema.

\begin{tabular}{|c|l|}
\hline Actor & \multicolumn{1}{|c|}{ Descripción } \\
\hline Administrador & $\begin{array}{l}\text { Se encarga de administrar el sistema, } \\
\text { gestionar los usuarios. }\end{array}$ \\
$\begin{array}{c}\text { Asesor de } \\
\text { Gestión de } \\
\text { Proyectos }\end{array}$ & $\begin{array}{l}\text { Es el encargado de asesorar, monitorear y } \\
\text { controlar la información suministrada por } \\
\text { los jefes de proyectos así como los reportes } \\
\text { correspondientes a los informes antes } \\
\text { mencionados. }\end{array}$ \\
\hline Experto & $\begin{array}{l}\text { Es el encargado de evaluar el proyecto para } \\
\text { ser aprobado (Dictamen de Evaluación). }\end{array}$ \\
\hline $\begin{array}{c}\text { Jefe de } \\
\text { proyecto }\end{array}$ & $\begin{array}{l}\text { Es el encargado llenar los datos del Perfil } \\
\text { de Proyecto, Ficha de Resultado, Informe } \\
\text { Semestral, Informe Final de su proyecto en el } \\
\text { sistema. }\end{array}$ \\
\hline Invitado & $\begin{array}{l}\text { Solo puede visualizar la convocatoria y } \\
\text { documentos y legislaciones ministeriales. }\end{array}$ \\
\hline
\end{tabular}

Fuente: Elaboración Propia (2016)

\section{Modelo de Datos}

El modelo de datos describe una representación de los datos persistentes del sistema. Para esto es necesario analizar cuáles son las clases persistentes, aquellas capaces de mantener su valor en el espacio y el tiempo, es decir, las que serán almacenadas en la base de datos.
El diseño y construcción de la Base de Datos (BD) se realizó con las clases persistentes identificadas. Por a la necesidad de que los asesores de gestión de proyecto tengan un mejor control de los recursos humanos participantes y evitar que un mismo investigador registre su nombre de varias maneras en diferentes proyectos, se hace necesario relacionar la BD del sistema con los datos del SIGENU ${ }^{2}$ y del ASSET $^{3}$. Lo anterior está dado a que estos sistemas no permiten acceder directamente a estas bases de datos. Esta relación es llevada a través de ficheros Excel facilitados por los administradores de estos sistemas y mediante una función PHP esta información pasa a formar parte de las tablas correspondientes en BD del sistema.

\section{Descripción del sistema}

Para ingresar el sistema es necesario registrarse, en la interfaz "Nuevo Usuario" (Figura 2). Es necesario en este paso determinar el rol dentro del sistema. En el caso de los roles asesor, y experto es necesaria la autorización del administrador del sistema. Una vez creado el usuario nuevo el sistema lo mostrará en la lista de la interfaz "Gestión de Usuario".

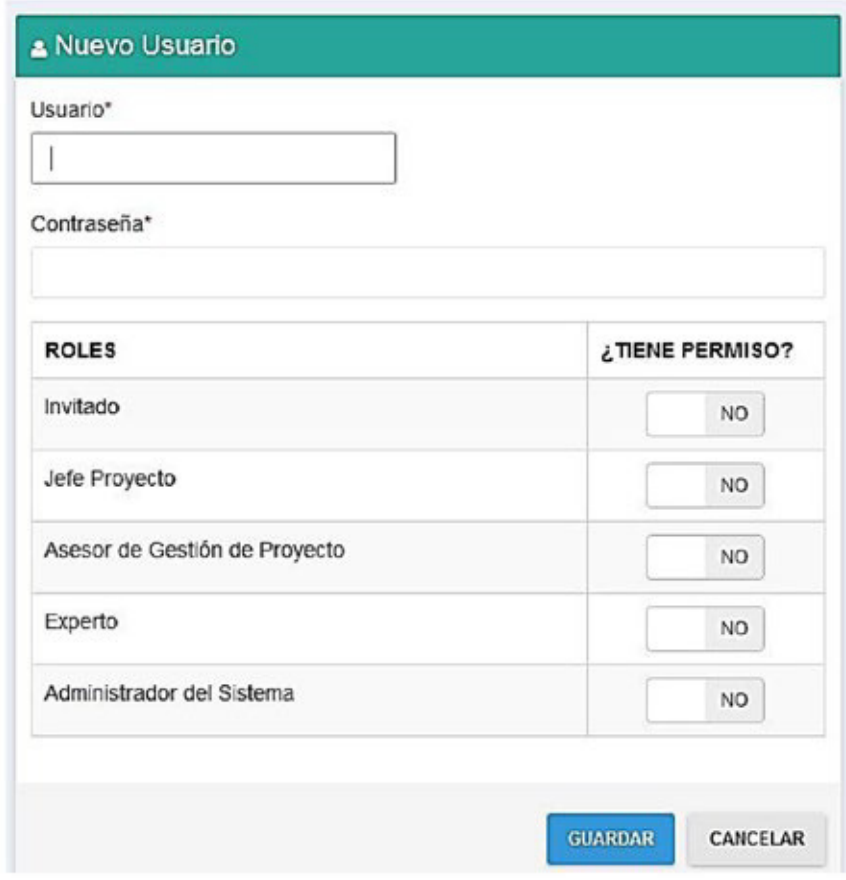

Figura 2 Interfaz del sistema "Nuevo Usuario"

Una de las ventajas que ofrece este sistema es al ingresar la información de los miembros del proyecto (Figura 3), debido a que el sistema maneja los datos de la base de datos del ASSET y SIGENU, lo cual permite 
autocompletar nombre y apellidos del investigador, su categoría docente, científica o académica y área a la cual pertenece. En el caso de ser un estudiante permite autocompletar el año académico y la carrera que cursa. Lo anterior facilita la actualización de la información del potencial científico en caso de cambio de categoría científica, académica o docente, movimiento de área de trabajo o en caso de causar una baja algún trabajador o estudiante. Esta funcionalidad es ejecutada por el administrador del sistema, que accede a ella a través del botón Administrador.

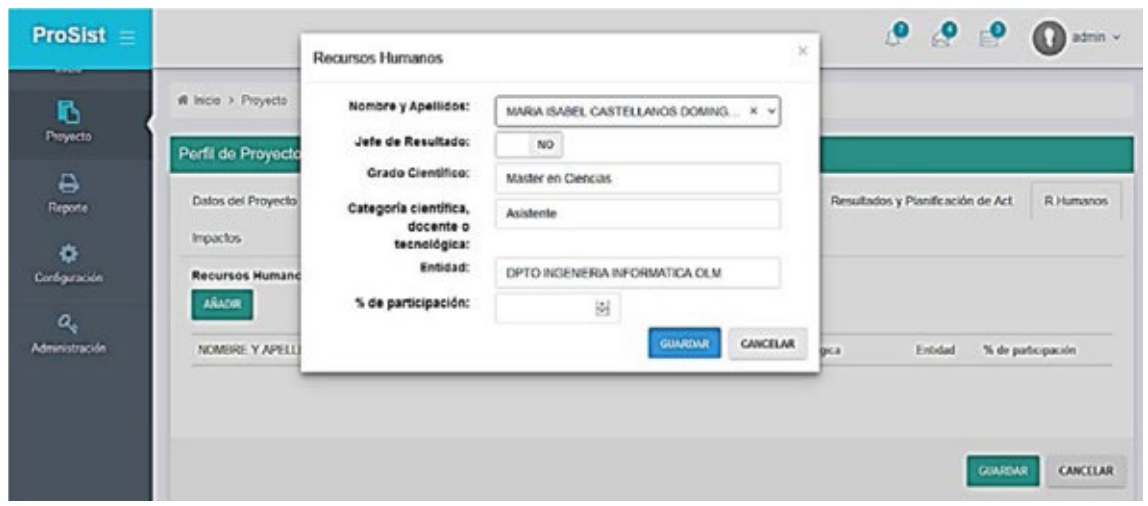

Figura 3. Interfaz del sistema "Recursos Humanos"

Para la creación del perfil del proyecto (Figura 4), el sistema permite autocompletar un formulario con los datos generales del proyecto donde el jefe de proyecto tiene que escoger de las clasificaciones que el sistema ofrece como por ejemplo prioridad, tipo de proyecto y clasificación. Esta interfaz es desplegada cuando el jefe de proyecto hace clic en el botón Crear de la interfaz Proyecto.

Figura 3. Interfaz del sistema "Perfil del proyecto"

\begin{tabular}{|c|c|c|c|c|c|c|c|c|}
\hline \multicolumn{9}{|c|}{ Perfil de Proyecto (Anexo 2) } \\
\hline Datos del Proyecto & Usuarios o Clientes & Problema a Resoher & \multirow[t]{2}{*}{ Objetivos } & \multicolumn{2}{|c|}{ Metodologia a Utizar } & \multicolumn{3}{|c|}{ Resultados y Planificación de Act. } \\
\hline R.Humanos Impacto & \multicolumn{7}{|c|}{ Impactos } & \\
\hline $\begin{array}{l}\text { Titulo del } \\
\text { Programa: }\end{array}$ & & $v$ & Código:" & & \& & Múmero: & & 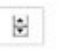 \\
\hline \multicolumn{9}{|l|}{$\begin{array}{l}\text { Titulo del } \\
\text { Proyecto: }\end{array}$} \\
\hline Clasificación:" & & $\nabla$ & \multicolumn{2}{|c|}{ Tipo Proyecto: } & \multicolumn{4}{|c|}{$\checkmark$} \\
\hline Jele Proyecto: & Select & $\checkmark$ & \multicolumn{2}{|c|}{ Fecha Inicio: } & Jurio & $v$ & 2017 & $\div$ \\
\hline \multirow[t]{2}{*}{ Entidad Principal: } & Select & $\checkmark$ & \multirow{2}{*}{\multicolumn{2}{|c|}{$\begin{array}{r}\text { Fecha } \\
\text { Terminaciôn:" }\end{array}$}} & Ociemb & $v$ & 2020 & t \\
\hline & Ensdad Ejecutora Pr & cipal & & & & & & \\
\hline \multicolumn{9}{|l|}{ Prioridad:" } \\
\hline & \multicolumn{8}{|c|}{ Prioricad Nadionaimente Establecica a la que responde o de interés insthucional o Empresarial } \\
\hline $\begin{array}{r}\text { Entidad } \\
\text { Participante: }\end{array}$ & & & & & & & & \\
\hline
\end{tabular}

El sistema consta de un panel izquierdo donde se agrupan las diferentes facilidades que el sistema debe permitir y un espacio de trabajo donde los usuarios pueden interactuar con el sistema. Dentro de las facilidades que ofrece a todos los usuarios está una lista con todos proyectos y los datos generales de este para que cualquier investigador interesado en pertenecer al proyecto o establecer cualquier colaboración sepa las líneas de investigación de la UHo y pueda contactar con los jefes de proyectos.

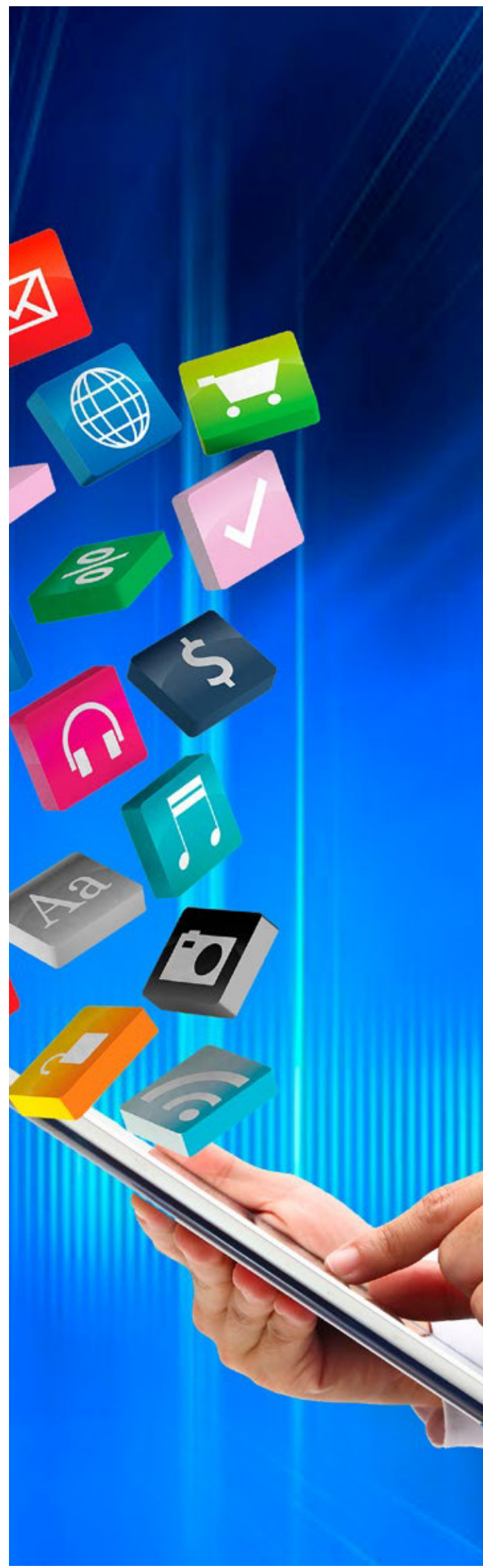




\section{CONCLUSIONES}

El número de proyectos que se maneja en DCTI de la UHo genera grandes volúmenes de información por lo que se requiere de una adecuada organización, monitoreo y control por parte de los asesores de proyectos, razón por la cual se requiere informatizar lo que facilita la gestión de la información de este proceso.

El vínculo cliente-desarrollador en el proceso iterativo e incremental que ofrece la metodología de desarrollo de software Iconix, permitió una mayor adecuación a las necesidades del cliente y maximizar los índices de calidad del sistema informático resultante.

El sistema informático desarrollado facilita el trabajo tanto de los asesores de proyecto de la DCTI como los jefes de proyectos, debido a que se tiene bien estructurada toda la información de los proyectos de I+D+i lo cual apoya la planificación, monitoreo y control de este proceso y facilita la toma de decisiones al respecto.

La generación de reportes en correspondencia a los modelos de las Indicaciones Metodológicas de la Resolución 44 del CITMA permite una estandarización en la información de los proyectos lo que permite obtener de forma automática datos estadísticos como el potencial científico en proyectos entre otros. Esto conlleva a una mayor eficiencia de los asesores de proyectos al entregar los informes solicitados por las Vicerrectorías y Rectoría.

ProSist es un sistema informático con amplia capacidad de generalización debido a que fue diseñado bajo estricto cumplimiento de los modelos exigidos por las Indicaciones Metodológicas de la Resolución 44 del CITMA por lo que puede ser utilizado en cualquier entidad que gestione

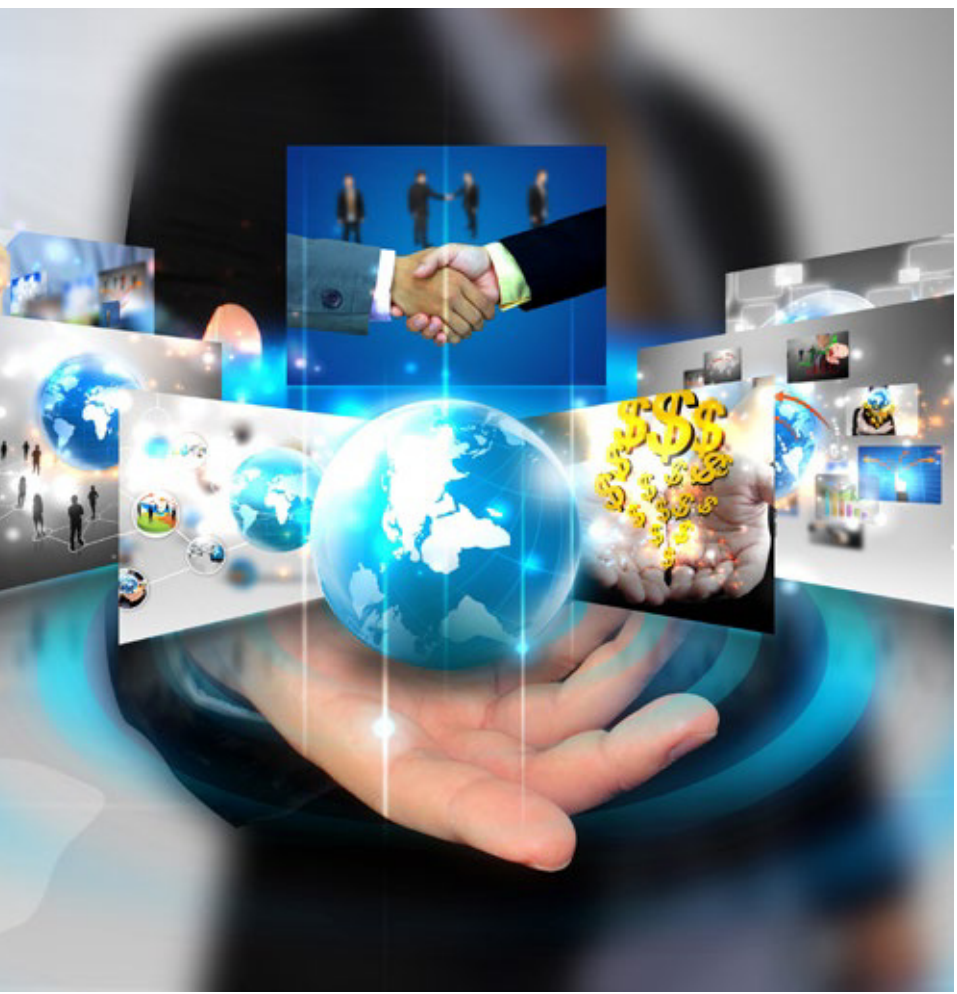

proyectos de investigación en Cuba. Además puede ser utilizado en las delegaciones provinciales del CITMA para la gestión de la información de los proyectos del territorio a un nivel macro.

\section{BIBILIOGRAFÍA}

[1] Armand, S., Extending Symfony2 Web Application Framework. 2014: Packt Publishing Ltd.

[2] Cabello, M.V.N., Introducción a las bases de datos relacionales. 2010: Editorial Visión Libros.

[3] Gauchat, J.D., HTML5 para Mentes Maestras, 2da Edición: Cómo aprovechar HTML5 para crear sitios web adaptables y aplicaciones revolucionarias. 2017: JD Gauchat.

[4] Gandhi, N., et al. Mimo control of an apache web server: Modeling and controller design. in American Control Conference, 2002. Proceedings of the 2002. 2002. IEEE.

[5] Gutiérrez, J.J., ¿ Qué es un framework web? Available in: http:// www.Isi.us.es/ javierj/investigacion_ficheros/Framework. pdf 2014.

[6] Heurtel, O., PHP 5.6: desarrollar un sitio web dinámico e interactivo. 2015: Ediciones ENI.

[7] Hoffman, S., M. Benton, and C. Java, Javascript: The Ultimate Guide to Javascript Programming and Computer Hacking (javascript for beginners, how to program, hacking exposed, hacking, how (Volume 12). 2016.

[8] Laguna Cruz, J.A., M.I. Castellanos Domínguez, and N.I. León Pupo. Perfeccionamiento de la Gestión de Proyectos de Ciencia, Tecnología e Innovación. Experiencias Universidad de Holguín. in Conferencia Científica Internacional. Octava Edición. 2017.

[9] León Pupo, N.I., Sistema Informativo para la Dirección de Ciencia y Técnica (DCT) en la Universidad de Holguín (UHo). 2016: Holguín.

[10] Martín, A.R. and M.J.R. Martín, Aplicaciones Web. 2014: Ediciones Paraninfo, SA.

[11] Murray, S., Interactive Data Visualization for the Web: An Introduction to Designing with. 2017: « O»Reilly Media, Inc.».

[12] Ministerio de Ciencia Tecnología y Medioambiente, Resolución 44/2012 Reglamento para el proceso de elaboración, aprobación, planificación, ejecución y control de los programas y proyectos de ciencia, tecnología e innovación. 2012, Gaceta Oficial de la República de Cuba: Ciudad de la Habana, Cuba.

[13] Ministerio de Ciencia Tecnología y Medio Ambiente, C., Indicaciones metodológicas para la actividad de programas y proyectos de ciencia, tecnología e innovación CITMA, Editor. 2014: La Habana.

[14] Nixon, R., Learning PHP, MySQL, JavaScript, CSS \& HTML5: A Step-by-Step Guide to Creating Dynamic Websites. 2014: « O)Reilly Media, Inc.».

[15] Ortíz Pérez, A., Tecnología para la gestión integrada de procesos en las universidades. Aplicación en la Universidad de Holguín, in Ingeniería Industrial. 2014, Universidad de Holguín: Holguín. p. 190.

[16] Olsson, M., Using PHP, in PHP 7 Quick Scripting Reference. 2016, Springer. p. 1-4.

[17] Rosenberg, D., M. Stephens, and M. Collins-Cope, Agile development with ICONIX process. NY: Apress, 2005.

[17] Stoner, J.A.F., R.E. Freeman, and D.R. Gilbert, Administración. 1996: Pearson educación.

[19] Valle, J.G. and J.G. Gutierrez, Definición arquitectura cliente servidor. Obtenido de Monografías. com: http://www. monografias. com/trabajos24/arquitecturacliente-servidor/ arquitectura-cliente-servidor. shtml, 2005. 